\title{
Multiaxial fatigue assessment of welded steel details according to the peak stress method based on tetra elements
}

\author{
Alberto Campagnolo ${ }^{1}$, Giovanni Meneghetti ${ }^{1,1}$, Vittorio Babini ${ }^{2}$, Matteo Riboli ${ }^{3,4}$ and \\ Andrea Spagnoli ${ }^{4}$ \\ ${ }^{1}$ University of Padova, Department of Industrial Engineering, 35131 Padova, Italy \\ ${ }^{2}$ Zamperla Spa, 36077 Altavilla Vicentina, Italy \\ ${ }^{3}$ Limitorque Fluid Power System, Flowserve S.r.l., 29122 Piacenza, Italy \\ ${ }^{4}$ University of Parma, Department of Engineering and Architecture, 43124 Parma, Italy
}

\begin{abstract}
The Peak Stress Method (PSM) is an engineering, FE-oriented application of the notch stress intensity factor (NSIF) approach to fatigue design of welded joints, which takes advantage of the singular linear elastic peak stresses from FE analyses with coarse meshes. Originally, the PSM was calibrated by using $3 \mathrm{D}$, eight-node brick elements, taking advantage of the submodeling technique. Recently, the PSM has been calibrated by using tennode tetra elements, which are able to directly discretize complex 3D geometries without the need for submodels. The PSM was validated for pure axial or bending loadings as well as pure torsion loadings; recently it has been extended to multiaxial loadings by adopting a design stress, the socalled equivalent peak stress, in conjunction with a reference design fatigue curve. After having briefly recalled the calibration of the PSM with tetra elements, the paper presents some applications of the PSM relevant to steel plate-to-tube welded details of industrial interest under in-phase bendingtorsion fatigue loadings. Experimental data have been re-analysed using the PSM based on tetra elements. Eventually a good agreement between experimental and theoretical results has been obtained in terms of fatigue crack initiation location as well as total fatigue life.
\end{abstract}

\section{Introduction}

In the fatigue design of welded joints, the approaches based on Notch Stress Intensity Factors (NSIF) assume both the weld toe and the weld root as sharp V-notches, i.e. with a notch tip radius $\rho=0$ (worst case hypothesis) and notch opening angles typically equal to $135^{\circ}$ and $0^{\circ}$, respectively $[1,2]$. Then singular, linear elastic stress fields in the vicinity of the 
notch tip can be quantitatively described by means of the relevant NSIFs, which can be defined according to Gross and Mendelson [3] by means of Eq. (1).

$\mathrm{K}_{\mathrm{i}}=\sqrt{2 \pi} \cdot \lim _{\mathrm{r} \rightarrow 0}\left\lfloor\left(\sigma_{\mathrm{jk}}\right)_{\theta=0} \cdot \mathrm{r}^{1-\lambda_{\mathrm{i}}}\right\rfloor \quad$ where $\mathrm{i}=1,2,3 \quad$ and $\quad \sigma_{\mathrm{jk}}=\sigma_{\theta \theta}, \tau_{\mathrm{r} \theta}, \tau_{\theta z}$ respectively

In previous expression, $\lambda_{\mathrm{i}}$ is the stress singularity exponent tied to mode I, II and III for $\mathrm{i}$ $=1,2$ and 3 , respectively, which depends on the notch opening angle $2 \alpha$, while the stress components $\sigma_{\theta \theta}, \tau_{\mathrm{r} \theta}$ and $\tau_{\theta z}$ are calculated along the notch bisector line $(\theta=0$ in Fig. 1a).

(a)

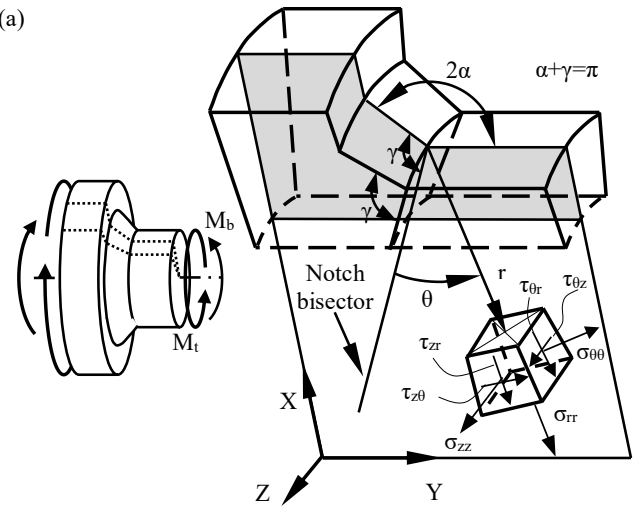

(b)

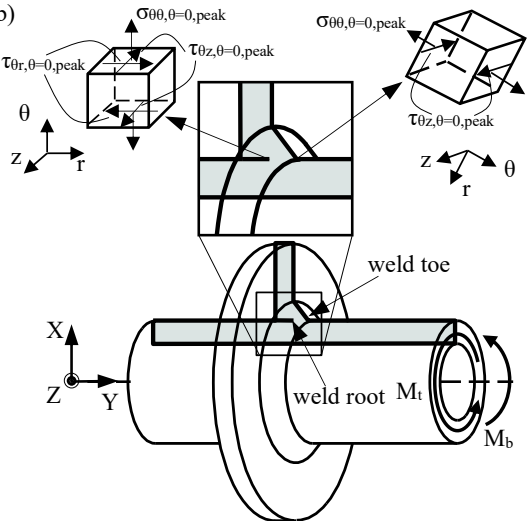

Fig 1: (a) Polar reference system centred at the weld toe of a typical tube-to-flange welded joint geometry subjected to multiaxial bending and torsion loading. (b) Sharp V-shaped notches in a welded joint at the weld root $\left(2 \alpha=0^{\circ}\right)$ and at the weld toe $\left(2 \alpha\right.$ typically equal to $\left.135^{\circ}\right)$ sides. Definition of peak

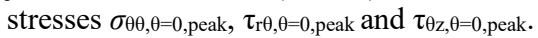

Lazzarin et al. [4] assumed the strain energy density (SED) averaged over a structural volume surrounding the weld root or the weld toe as a fatigue strength criterion. They assumed a structural volume having circular shape with radius $\mathrm{R}_{0}$ and provided the closedform expression of the averaged SED parameter as a function of the relevant NSIFs. Dealing with a general multiaxial fatigue loading condition (mixed mode I+II+III loading, see Fig. 1), the SED averaged over the control volume can be expressed as follows [4]:

$\Delta \overline{\mathrm{W}}=\mathrm{c}_{\mathrm{w} 1} \frac{\mathrm{e}_{1}}{\mathrm{E}}\left[\frac{\Delta \mathrm{K}_{1}}{\mathrm{R}_{0}{ }^{1-\lambda_{1}}}\right]^{2}+\mathrm{c}_{\mathrm{w} 2} \frac{\mathrm{e}_{2}}{\mathrm{E}}\left[\frac{\Delta \mathrm{K}_{2}}{\mathrm{R}_{0}^{1-\lambda_{2}}}\right]^{2}+\mathrm{c}_{\mathrm{w} 3} \frac{\mathrm{e}_{3}}{\mathrm{E}}\left[\frac{\Delta \mathrm{K}_{3}}{\mathrm{R}_{0}^{1-\lambda_{3}}}\right]^{2}$

where $\mathrm{E}$ is the Young's modulus, $\mathrm{e}_{1}, \mathrm{e}_{2}$ and $\mathrm{e}_{3}$ are known parameters which depend on the notch opening angle $2 \alpha$ and on the Poisson's ratio $v$ [4], while $\Delta \mathrm{K}_{1}, \Delta \mathrm{K}_{2}$ and $\Delta \mathrm{K}_{3}$ are the range values of the NSIFs relevant to mode I, II and III, respectively. The control radius $R_{0}$ was calibrated considering arc-welded joints made of structural steel and it was found to be $0.28 \mathrm{~mm}$ [5]. Finally, the coefficients $c_{\mathrm{wi}}(\mathrm{i}=1,2,3$ indicates the loading mode) depend on the nominal load ratio $R$ according to the following expression [6]:

$c_{w}(R)=\left\{\begin{array}{lll}\frac{1+R^{2}}{(1-R)^{2}} & \text { if } & -1 \leq R \leq 0 \\ \frac{1-R^{2}}{(1-R)^{2}} & \text { if } & 0<R<1\end{array}\right.$ 
In particular, the parameter $c_{\mathrm{w}}$ equals 0.5 for $R=-1$ and 1 for $R=0$. It should be noted that welded joints loaded in the as-welded conditions are almost not sensitive to mean stresses, according to design standards [7], therefore Eq. (2) with $c_{\mathrm{wi}}=1$ should be applied.

Nevertheless, it should be noted that the calculation of NSIFs on the basis of the results of numerical analyses shows a major drawback in engineering applications, since very refined FE meshes (element size on the order of $10^{-5} \mathrm{~mm}$ ) are required in order to apply definition (1) to calculate the NSIFs. When dealing with three-dimensional notched components, both the solution of the FE model and the post-processing of numerical results could be even more time-consuming. On the other hand, Lazzarin and co-workers underlined that the averaged SED can be calculated directly by FEM adopting coarse meshes within the control volume characterized by a radius $R_{0}$ [8]. Alternatively, the Peak Stress Method (PSM) may be used to estimate the NSIFs by FEM adopting even coarser meshes [9] without modelling the control volume. Moreover, the PSM requires only the singular, linear elastic peak stresses evaluated at the $\mathrm{V}$-notch tip, instead of a number of stress-distance numerical results, as required in order to calculate NSIFs on the basis of their definitions, Eq. (1).

\section{The Peak Stress Method (PSM)}

The PSM is a rapid technique to estimate approximately the NSIFs $\mathrm{K}_{1}, \mathrm{~K}_{2}$ and $\mathrm{K}_{3}$ from the singular, linear elastic, opening, sliding and anti-plane FE peak stresses $\sigma_{\theta \theta, \theta=0 \text {,peak, }}$ $\tau_{\mathrm{r} \theta, \theta=0 \text {,peak }}$ and $\tau_{\theta z, \theta=0 \text {,peak, }}$, respectively, which are referred to the $\mathrm{V}$-notch bisector line (see the example in Fig. 1b), and calculated at the V-notch tip from FE analyses with coarse meshes. The estimated NSIF values can be obtained from the following expressions [9-11]:

$$
\mathrm{K}_{1} \cong \mathrm{K}_{\mathrm{FE}}^{*} \cdot \sigma_{\theta \theta, \theta=0, \text { peak }} \cdot \mathrm{d}^{1-\lambda_{1}} ; \quad \mathrm{K}_{2} \cong \mathrm{K}_{\mathrm{FE}}^{* *} \cdot \tau_{\mathrm{r} \theta, \theta=0, \text { peak }} \cdot \mathrm{d}^{0.5} ; \quad \mathrm{K}_{3} \cong \mathrm{K}_{\mathrm{FE}}^{* * *} \cdot \tau_{\theta z, \theta=0, \text { peak }} \cdot \mathrm{d}^{1-\lambda_{3}}
$$

where $d$ is the so-called 'global element size', i.e. the average FE size adopted by the free mesh generation algorithm available in the numerical software. Parameters $\mathrm{K}_{\mathrm{FE}}^{*}, \mathrm{~K}_{\mathrm{FE}}^{* *}$ and $\mathrm{K}_{\mathrm{FE}}^{* * *}$ depend on the calibration options: (i) element type and formulation; (ii) mesh pattern of finite elements and (iii) procedure for stress extrapolation at FE nodes. Originally, the PSM for 3D models has been calibrated by using eight-node brick elements [12], taking advantage of the submodeling technique available in Ansys ${ }^{\circledR}$ software. To speed up even more the application of PSM by means of 3D models, in a recent contribution [13] parameters $\mathrm{K}_{\mathrm{FE}}^{*}, \mathrm{~K}_{\mathrm{FE}}^{* *}$ and $\mathrm{K}_{\mathrm{FE}}^{* * *}$ have been calibrated by using ten-node tetra elements, which are able to discretize complex 3D geometries, making the PSM applicable directly to a single model, without the need for submodels.

\subsection{Calibrating the PSM with 10-node tetrahedral elements}

It has been shown in [13] that when adopting tetrahedral elements to analyse a 3D notch problem, the mesh pattern obtained by the free mesh generation algorithm is intrinsically not regular, so that a node belonging to the notch tip could be shared by a different number of elements having significantly different shape. Therefore, the peak stress could vary along the notch tip profile even in the case of a constant applied NSIF. Accordingly, to reduce the variability of the peak stress along the notch tip profile, an average peak stress value has been introduced in [13]; it has been defined at the generic node $\mathrm{n}=\mathrm{k}$ as the moving average on three adjacent vertex nodes, i.e. $\mathrm{n}=\mathrm{k}-1, \mathrm{k}$ and $\mathrm{k}+1$ :

$\bar{\sigma}_{\mathrm{ij}, \text { peak }, \mathrm{n}=\mathrm{k}}=\left.\frac{\sigma_{\mathrm{ij}, \text { peak }, \mathrm{n}=\mathrm{k}-1}+\sigma_{\mathrm{ij}, \text { peak }, \mathrm{n}=\mathrm{k}}+\sigma_{\mathrm{ij}, \text { peak }, \mathrm{n}=\mathrm{k}+1}}{3}\right|_{\mathrm{n}=\text { node }}$ 
It is worth noting that only peak stress values calculated at vertex nodes of the quadratic tetrahedral elements have to be input in Eq. (5), i.e. stress values at mid-side nodes located at the notch tip profile must be neglected. Given this, the PSM parameters $\mathrm{K}_{\mathrm{FE}}^{*}, \mathrm{~K}^{* *} \mathrm{FE}$ and $\mathrm{K}_{\mathrm{FE}}^{* * *}$ have been calibrated in [13] by applying Eq. (4), re-arranged as Eq. (6), to several 3D notch problems under pure mode I, pure mode II and pure mode III loadings analysed adopting 3D, ten-node, quadratic tetrahedral elements (SOLID 187 of Ansys ${ }^{\circledR}$ library):

$\mathrm{K}_{\mathrm{FE}}^{*} \cong \frac{\mathrm{K}_{1}}{\bar{\sigma}_{\theta \theta, \theta=0, \text { peak }} \cdot \mathrm{d}^{1-\lambda_{1}}} ; \quad \mathrm{K}_{\mathrm{FE}}^{* *} \cong \frac{\mathrm{K}_{2}}{\bar{\tau}_{\mathrm{r} \theta \theta=0, \text { peak }} \cdot \mathrm{d}^{0.5}} ; \quad \mathrm{K}_{\mathrm{FE}}^{* * *} \cong \frac{\mathrm{K}_{3}}{\bar{\tau}_{\theta z, \theta=0, \text { peak }} \cdot \mathrm{d}^{1-\lambda_{3}}}$

The results obtained from the calibration of the 3D-PSM based on ten-node tetra elements are summarised in Table 1 , along with the minimum mesh density ratios $a / d$, $a$ being the characteristic size of the considered sharp V-notch, which guarantee the convergence of the PSM parameters $\mathrm{K}_{\mathrm{FE}}^{*}, \mathrm{~K}_{\mathrm{FE}}^{* *}$ and $\mathrm{K}_{\mathrm{FE}}^{* * *}$, respectively.

Table 1: Summary of calibration of $\mathrm{K}_{\mathrm{FE}}^{*}, \mathrm{~K}_{\mathrm{FE}}^{* *}$ and $\mathrm{K}^{* * *} \mathrm{FE}$ for 10-node tetrahedral elements [13].

\begin{tabular}{|c|c|c|c|c|c|c|}
\hline \multirow{2}{*}{$\begin{array}{l}2 \alpha \\
{\left[{ }^{\circ}\right]}\end{array}$} & \multicolumn{2}{|l|}{ Mode I } & \multicolumn{2}{|l|}{ Mode II } & \multicolumn{2}{|l|}{ Mode III } \\
\hline & $\mathbf{K}_{\mathrm{FE}}^{*}$ & $(a / d)_{\min }$ & $\mathbf{K}^{* *} \mathrm{FE}$ & $(a / d)_{\min }$ & $\mathbf{K}^{* * * *} \mathrm{FE}$ & $(a / d)_{\min }$ \\
\hline 0 & $1.01 \pm 15 \%$ & 3 & $1.63 \pm 20 \%$ & 1 & $1.37 \pm 10 \%$ & 2 \\
\hline 90 & $1.01 \pm 15 \%$ & 3 & I & 1 & 1 & 1 \\
\hline 135 & $1.21 \pm 10 \%$ & 1 & I & 1 & $1.75 \pm 5 \%$ & 2 \\
\hline
\end{tabular}

\subsection{Defining a SED-based design stress using the PSM}

By using the PSM-based relationships (Eq. (6)), the closed-form expression of the averaged SED, Eq. (2), can be rewritten as a function of the singular, linear elastic FE average peak stresses $\bar{\sigma}_{\theta \theta \theta \theta=0, \text { pak }}, \quad \bar{\tau}_{\mathrm{r} \theta \theta \theta=0, \text { peak }}$ and $\bar{\tau}_{\theta z, \theta=0, \text { pak }}$. Then, by considering the equality $\mathrm{W}=\left(1-v^{2}\right) \cdot \sigma_{\text {eq,peak }}^{2} / 2 \mathrm{E}$ valid under plane strain conditions, the equivalent peak stress, $\sigma_{\text {eq,peak, }}$, can be derived:

$$
\begin{aligned}
& \Delta \overline{\mathrm{W}}=\mathrm{c}_{\mathrm{w} 1} \frac{\mathrm{e}_{1}}{\mathrm{E}}\left[\mathrm{K}_{\mathrm{FE}}^{*} \cdot \Delta \overline{\mathrm{\sigma}}_{\theta \theta \theta \theta=0, \text { peak }} \cdot\left(\frac{\mathrm{d}}{\mathrm{R}_{0}}\right)^{1 . \alpha_{1}}\right]^{2}+\mathrm{c}_{\mathrm{w} 2} \frac{\mathrm{e}_{2}}{\mathrm{E}}\left[\mathrm{K}_{\mathrm{FE}}^{* *} \cdot \Delta \bar{\tau}_{\mathrm{r} \theta \theta \theta 0, \text { peak }} \cdot\left(\frac{\mathrm{d}}{\mathrm{R}_{0}}\right)^{1 . \alpha_{2}}\right]^{2} \\
& +\mathrm{c}_{\mathrm{w} 3} \frac{\mathrm{e}_{3}}{\mathrm{E}}\left[\mathrm{K}_{\mathrm{FE}}^{* * *} \cdot \Delta \bar{\tau}_{\theta z, \theta=0, \text { peak }} \cdot\left(\frac{\mathrm{d}}{\mathrm{R}_{0}}\right)^{1 . x_{3}}\right]^{2}=\frac{1-\mathrm{v}^{2}}{2 \mathrm{E}} \Delta \sigma_{\text {eq,peak }}^{2}
\end{aligned}
$$

Afterward, the following expression is obtained for a general multiaxial loading condition:

$$
\Delta \sigma_{\text {eq,peak }}=\sqrt{\mathrm{c}_{\mathrm{w} 1} \cdot \mathrm{f}_{\mathrm{w} 1}^{2} \cdot \Delta \overline{\mathrm{\sigma}}_{\theta \theta, \theta=0, \text { peak }}^{2}+\mathrm{c}_{\mathrm{w} 2} \cdot \mathrm{f}_{\mathrm{w} 2}^{2} \cdot \Delta \bar{\tau}_{\mathrm{r} \theta, \theta=0, \text { peak }}^{2}+\mathrm{c}_{\mathrm{w} 3} \cdot \mathrm{f}_{\mathrm{w} 3}^{2} \cdot \Delta \bar{\tau}_{\theta \mathrm{z}, \theta=0, \text { peak }}^{2}}
$$

where the coefficients $f_{w 1}, f_{w 2}$ and $f_{w 3}$ are defined as following:

$$
\mathrm{f}_{\mathrm{wi}}=\mathrm{K}_{\mathrm{FE}} \cdot \sqrt{\frac{2 \mathrm{e}_{\mathrm{i}}}{1-v^{2}}} \cdot\left(\frac{\mathrm{d}}{\mathrm{R}_{0}}\right)^{1-\mathrm{x}_{\mathrm{i}}} \quad \text { where } \mathrm{i}=1,2,3
$$




\section{Structural steels joints of industrial interest: geometries and FE stress analyses according to the PSM based on tetra elements}

Plate-to-tube welded steel details have been fatigue tested in [14] under in-phase bendingtorsion fatigue loadings. The following industrial case studies have been considered: (i) fullpenetration joints (see model 1 in Table 2) both in the as-welded and stress-relieved conditions, which are adopted in the structure of a roundabout-carousel; (ii) fillet-welded joints (see model 2 in Table 2) in the stress-relieved conditions, which are present in a quarterturn scotch yoke valve actuators, typically used in oil \& gas, power and chemical industries. In the present manuscript, the experimental fatigue results generated in [14] will be reanalysed according to the 3D-PSM based on tetra elements (SOLID 187 of Ansys ${ }^{\circledR}$ element library). For the sake of brevity, only few details about the analysis procedure according to PSM are reported here, the reader being referred to [14] for additional details. Table 2 reports the joint geometries and details about FE analyses according to PSM.

\subsection{Full-penetration plate-to-tube joints (model 1 of Table 2)}

Table 2 shows that the FE model consists of only half of the specimen, since the symmetry on the YZ plane has been employed. Mode II stresses being not singular at the weld toe, the mesh density ratio must be $a / d \geq 2$ to satisfy the conditions of applicability of the PSM based on tetra elements at the weld toe under mode III loadings (see Table 1). The tube thickness being $a=8 \mathrm{~mm}$, then the average local element size $d_{\text {local }}$ has been set to $8 / 2=4 \mathrm{~mm}$, by locally refining a global FE mesh having element size $d_{\text {global }}=8 \mathrm{~mm}$ at the weld toe line (setting level = 1 and depth = 1 in Ansys FE code) and by repeating the refinement until the resulting local element size was $d_{\text {local }} \cong 4 \mathrm{~mm}$. After solving the FE model meshed using 10 node tetra elements, the peak stresses $\sigma_{\theta \theta, \theta=0 \text {,peak }}$ and $\tau_{\theta z, \theta=0, \text { peak }}$ have been calculated along the weld toe line; afterwards, the corresponding average values $\bar{\sigma}_{\theta \theta, \theta=0 \text {,peak }}$ and $\bar{\tau}_{\theta z, \theta=0 \text {,peak }}$ have been calculated according to Eq. (5) and have been reported in Fig. 2a as a function of the angular coordinate $\phi$. Then, the equivalent peak stress range has been evaluated from Eq. (8) and reported in the same figure. According to the PSM, the crack initiation location is anticipated at point $\mathrm{A}\left(\phi=0^{\circ}\right.$ in Fig. 2a), even if the equivalent peak stress is $10 \%$ lower than at point $\mathrm{B}$, because the mode I peak stress $\bar{\sigma}_{\theta \theta, \theta=0, \text { peak }}$ is tensile at point $A$, therefore it tends to open the initiated crack rather than close it. This prediction is in agreement with the experimental observations reported in [14].

\subsection{Fillet-welded scotch yoke joints (model 2 of Table 2)}

Table 2 shows that only half of the specimen geometry has been modelled since the YZ symmetry plane has been employed. To properly simulate the stress state due to the load cycle between $+\mathrm{F}$ and $-\mathrm{F}$, two different loading conditions were run, while Table 2 shows only the condition $+\mathrm{F}$ for sake of brevity (see [14] for more details). It can be observed that the most demanding condition to apply the PSM based on tetra elements is dictated by the mode I loading at the weld root side: according to Table 1 , the mesh density ratio must be $a / d \geq 3, a=5 \mathrm{~mm}$ being the weld leg length. Therefore, the adopted local average element size $d_{\text {local }}$ has been set equal to $5 / 3 \cong 1.5 \mathrm{~mm}$. This size has been obtained by locally refining a global FE mesh having element size $d_{\text {global }}=3 \mathrm{~mm}$ at the weld root and toe lines (setting level $=1$ and depth $=1$ in Ansys FE code) and by repeating the refinement until the resulting local element size was $d_{\text {local }} \cong 1.5 \mathrm{~mm}$. The peak stresses $\sigma_{\theta \theta, \theta=0, \text { peak }}, \tau_{\mathrm{r} \theta, \theta=0, \text { peak }}$ and $\tau_{\theta z, \theta=0, \text { peak }}$ have been calculated from both FE models (with loads $+F$ and $-F$, respectively, see [14]) 
along the root, wing-toe and tube-toe lines. Then, the corresponding average values $\bar{\sigma}_{\theta \theta, \theta=0 \text {,peak }}$ , $\bar{\tau}_{\mathrm{r} \theta, \theta=0, \text { peak }}$ and $\bar{\tau}_{\theta z, \theta=0 \text {,peak }}$ have been calculated according to Eq. (5). Finally, the equivalent peak stress range has been evaluated from Eq. (8) and reported in Fig. 2b. Figure $2 b$ shows that the absolute maximum value of the equivalent peak stress occurs along the weld root line at an angle $\phi$ of about $5^{\circ}$. Also in this case, the fatigue crack initiation location estimated from the PSM based on tetra elements is in agreement with the experimental evidences reported in [14].

Table 2: Joint geometries and FE analyses for fatigue strength assessment according to the PSM

\begin{tabular}{llll}
\hline Model & Joint Geometry - Loading Conditions & FE analyses \\
Model 1 \\
joint
\end{tabular}

\section{Model 2 \\ scotch yoke joint}

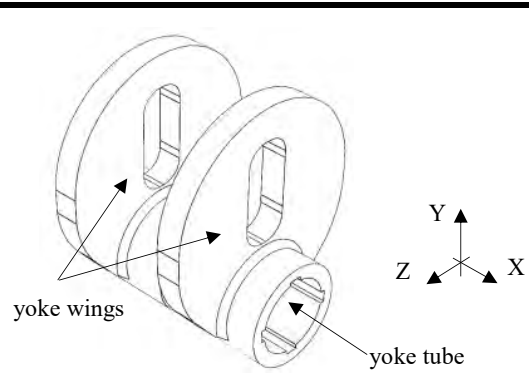

region where the applied load

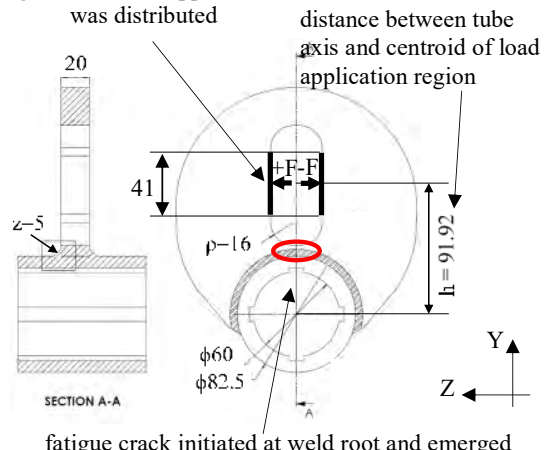

fatigue crack initiated at weld root and emerged at weld bead surface
Mainmodel Tetra-10 (SOLID187) $\mathrm{d}_{\text {global }}=3 \mathrm{~mm}$ constrained regions

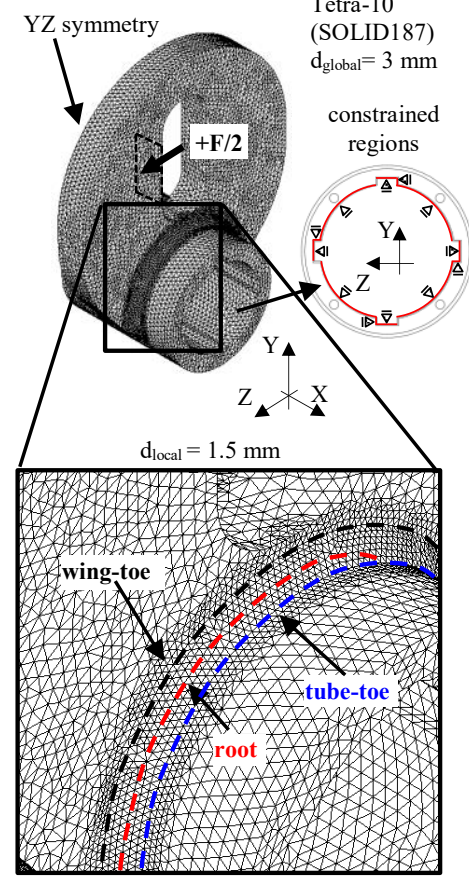


Finally, Fig. 2 shows that the equivalent peak stress results obtained by applying the PSM based on tetra elements match those previously obtained in [14] by adopting the PSM based on brick elements, i.e. taking advantage of the submodelling technique, the maximum deviation being about $10 \%$ at the root side of the scotch yoke joints (see Fig. 2b).
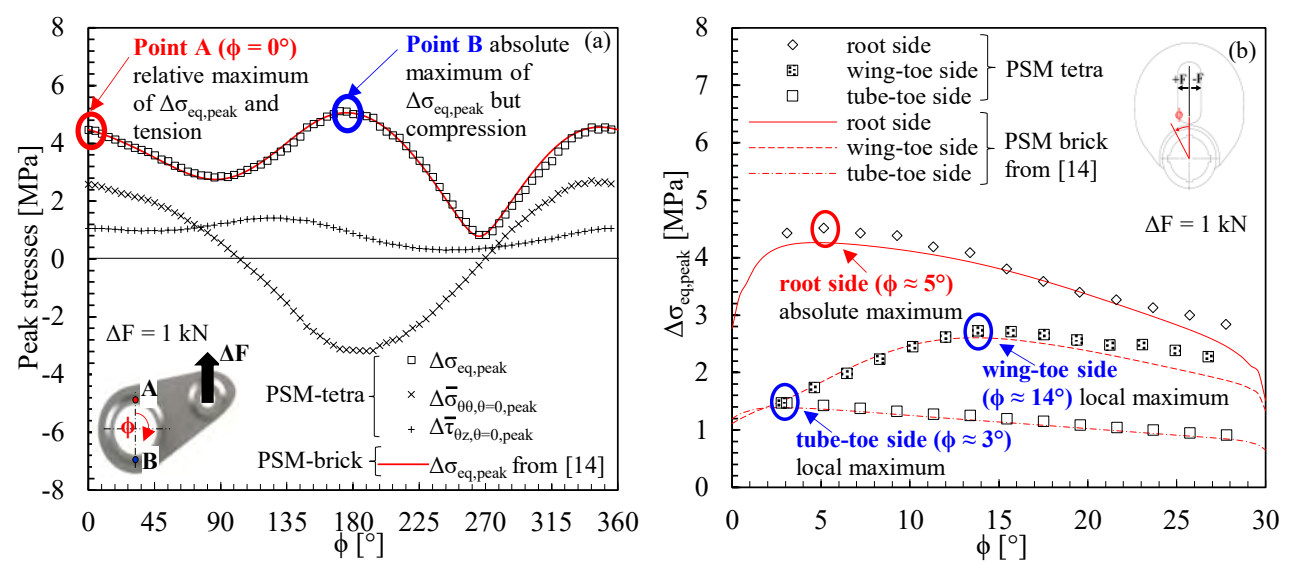

Fig. 2: Estimation of the crack initiation point in (a) full-penetration welded joints (model 1 of Table 2) and (b) fillet-welded joints (model 2 of Table 2) based on the distribution of the equivalent peak stress along the weld toe and root lines.

\section{Assessment of weld toe and weld root fatigue failures}

After applying the PSM based on tetra elements, the original experimental results [14] have been converted in terms of range of equivalent peak stress calculated at the point of crack initiation. To select the proper PSM-based design curve, the contribution of shear as compared to normal stresses must first be evaluated. To this aim, a local biaxiality ratio $\lambda_{\text {local }}$ has been defined in [14] as the ratio between the energy contributions tied to the shear modes of loading, i.e. mode II and III, and the energy contribution tied to the opening mode, i.e. mode I. The ratio $\lambda_{\text {local }}$ can be expressed as a function of the average peak stresses $\bar{\tau}_{\mathrm{r} \theta \theta=0, \text { pak }}$, $\bar{\tau}_{\theta z, \theta=0, \text { peak }}$ and $\bar{\sigma}_{\theta \theta, \theta=0, \text { peak }}$, which have been defined in Eq. (5), as follows:

$\lambda_{\text {local }}=\frac{\mathrm{f}_{\mathrm{w} 2}^{2} \cdot \Delta \bar{\tau}_{\mathrm{r} \theta \theta=0, \text { peak }}^{2}+\mathrm{f}_{\mathrm{w} 3}^{2} \cdot \Delta \bar{\tau}_{\theta \mathrm{z}, \theta=0, \text { peak }}^{2}}{\mathrm{f}_{\mathrm{w} 1}^{2} \cdot \Delta \bar{\sigma}_{\theta \theta, \theta=0, \text { peak }}^{2}}$

In the case of full-penetration joints, the ratio $\lambda_{\text {local }}$ calculated at the fatigue crack initiation location, i.e. $\phi=0$ at weld toe, is rather small (see [14] for a more detailed discussion) and equal to 0.40, according to the results reported in Fig. 2a. Accordingly, the experimental results have been compared in Fig. 3a with the PSM-based design scatter band previously calibrated [15] on experimental fatigue data generated from steel welded joints subjected to pure mode I loading (inverse slope $\mathrm{k}=3$ ). It can be observed from Fig. 3a that the design scatter band is slightly on the safe side especially in the high-cycle-fatigue regime. However, it has been shown in [14] that the adopted failure criterion, which has been defined at a 0.1 $\mathrm{mm}$ increase of the maximum displacement or equivalently at a $10 \%$-stiffness-drop, corresponded to a through thickness crack having surface length about equal to $70 \mathrm{~mm}$ according to both experimental and numerical results.

Concerning fillet-welded joints, according to the results of Fig. $2 b$, the ratio $\lambda_{\text {local }}$ equals 11.4 at the fatigue crack initiation point. The value 11.4 being rather high (see again [14]), the contribution due to mode III is predominant and, therefore, the experimental results have 
been compared in Fig. $3 \mathrm{~b}$ with the PSM-based design scatter band valid for steel welded joints subjected to pure mode III loading [11] $(\mathrm{k}=5)$. It can be observed from Fig. $3 \mathrm{~b}$ that the experimental results fall inside the PSM-based scatter band, which on the other hand exhibits a certain degree of conservatism. Also dealing with fillet-welded joints, it has been shown in [14] that the adopted failure criterion corresponded to a through crack having surface length about equal to $35 \mathrm{~mm}$ on the basis of numerical simulations.

Accordingly, the longer experimental fatigue lives than estimated by the PSM illustrated in both Figs. $3 a$ and $b$ might be explained because the adopted " 0.1 -mm-displacementincrease" criterion have led to fatigue cracks propagated well beyond the SED-structuralvolume dominated by the singular stress distributions, as it has been analysed and discussed thoroughly in [14]. Concerning this issue, more accurate experimental techniques for damage detection are beneficial to apply the PSM to complex welded joints exhibiting long crack propagation paths.
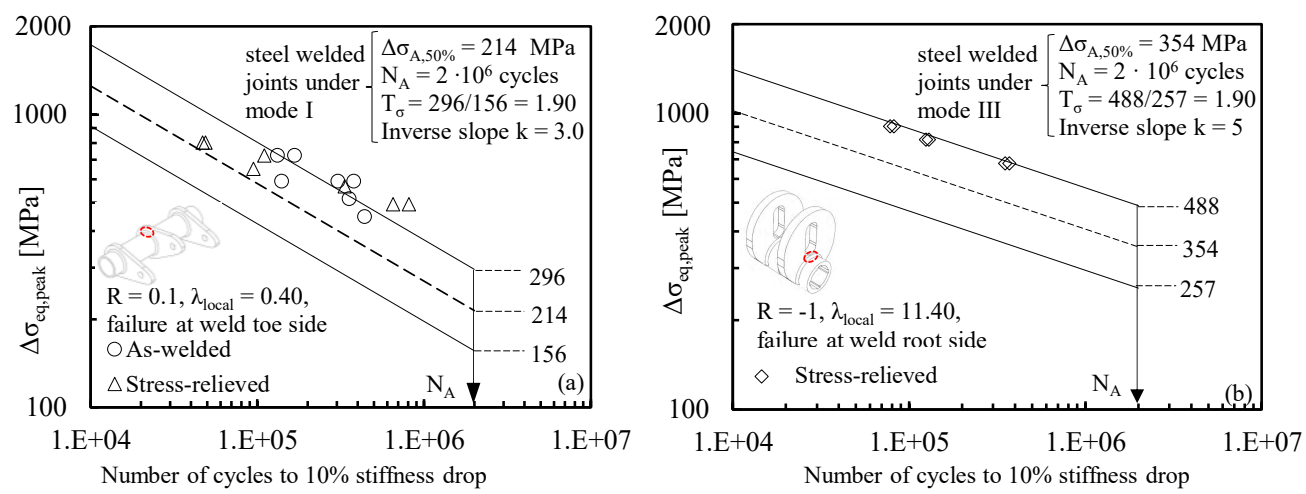

Fig. 3: Fatigue assessment of (a) toe failures in full-penetration structural steel welded joints (model 1 of Table 2) and (b) root failures in fillet-welded structural steel joints (model 2 of Table 2) under combined bending-torsion in-phase loading according to the PSM.

\section{Conclusions}

The peak stress method (PSM) employs the singular, linear elastic peak stresses evaluated at the toe and root sides by means of FE analyses with coarse meshes to rapidly estimate the mode I, mode II and mode III NSIFs. Recently, the 3D PSM has been calibrated by using ten-node tetra elements, which are able to discretize complex 3D geometries, making the PSM applicable directly without the need for submodels. By using the PSM in combination with the averaged Strain Energy Density (SED) as a fatigue strength criterion, a so-called equivalent peak stress has been defined to assess either weld toe or weld root fatigue failures in conjunction with a properly calibrated design curve. The PSM based on tetra elements has been applied to analyse weld toe as well as weld root fatigue failures generated in a recent contribution by testing plate-to-tube welded details in structural steel under in-phase multiaxial stresses. The equivalent peak stress results obtained by applying the PSM based on tetra elements match those previously obtained by adopting the PSM based on brick elements. Finally, the PSM based on tetra elements has proved to assess the fatigue crack initiation location in agreement with experimental observations, moreover a quite good agreement has been obtained between the experimental results and the PSM-based design scatter bands. 


\section{References}

1. P. Lazzarin, R. Tovo, A notch intensity factor approach to the stress analysis of welds, Fat. Fract. Eng. Mater. Struct., 21, 1089-1103. (1998)

2. D. Radaj, C.M. Sonsino, W. Fricke, Fatigue Assessment of Welded Joints by Local Approaches, Woodhead Publishing, Cambridge, (2006).

3. B. Gross, A. Mendelson, Plane elastostatic analysis of $V$-notched plates, Int. J. Fract. Mech., 8, 267-276 (1972)

4. P. Lazzarin, P. Livieri, F. Berto, M. Zappalorto, Local strain energy density and fatigue strength of welded joints under uniaxial and multiaxial loading, Eng. Fract. Mech., 75, 1875-1889 (2008)

5. P. Livieri, P. Lazzarin, Fatigue strength of steel and aluminium welded joints based on generalised stress intensity factors and local strain energy values, Int. J. Fract., 133, 247-276 (2005)

6. P. Lazzarin, C.M. Sonsino, R. Zambardi, A notch stress intensity approach to assess the multiaxial fatigue strength of welded tube-to-flange joints subjected to combined loadings, Fat. Fract. Eng. Mater. Struct., 27, 127-140 (2004)

7. Eurocode 3: Design of steel structures - part 1-9: Fatigue, CEN, (2005).

8. P. Lazzarin, F. Berto, M. Zappalorto, Rapid calculations of notch stress intensity factors based on averaged strain energy density from coarse meshes: Theoretical bases and applications, Int. J. Fat., 32, 1559-1567. (2010)

9. G. Meneghetti, P. Lazzarin, Significance of the elastic peak stress evaluated by FE analyses at the point of singularity of sharp V-notched components, Fat. Fract. Eng. Mater. Struct., 30, 95-106 (2007)

10. G. Meneghetti, The use of peak stresses for fatigue strength assessments of welded lap joints and cover plates with toe and root failures, Eng. Fract. Mech., 89, 40-51 (2012)

11. G. Meneghetti, The peak stress method for fatigue strength assessment of tube-toflange welded joints under torsion loading, Weld. World., 57, 265-275 (2013)

12. G. Meneghetti, C. Guzzella, The peak stress method to estimate the mode I notch stress intensity factor in welded joints using three-dimensional finite element models, Eng. Fract. Mech., 115 (2014).

13. A. Campagnolo, G. Meneghetti, Rapid estimation of notch stress intensity factors in $3 D$ large-scale welded structures using the peak stress method, MATEC Web Conf., 165 (2018).

14. G. Meneghetti, A. Campagnolo, V. Babini, M. Riboli, A. Spagnoli, Multiaxial fatigue assessment of welded steel details according to the peak stress method: industrial case studies, Int. J. Fat., 125, 362-380 (2019)

15. G. Meneghetti, P. Lazzarin, The Peak Stress Method for Fatigue Strength Assessment of welded joints with weld toe or weld root failures, Weld. World, 55 (2011). 